Material Behaviour

\title{
Construction of bi-layer biluminophore fast-responding pressure sensitive coating for non-contact unsteady aerodynamic testing
}

\author{
Di Yang ${ }^{\mathrm{a}}$, Junkai Ren ${ }^{\mathrm{b}}$, Jiwei $\mathrm{Li}^{\mathrm{c}}$, Qinglin Wang ${ }^{\mathrm{a}}$, Qiu Wang ${ }^{\mathrm{c}, *}$, Zheng Xie ${ }^{\mathrm{b},{ }^{, * *}}$, Xiaozhong Qu ${ }^{\mathrm{a}, * * * *}$ \\ ${ }^{a}$ Center of Materials Science and Optoelectronics Engineering, College of Materials Science and Opto-Electronic Technology, University of Chinese Academy of Sciences, \\ Beijing, 100049, China \\ ${ }^{\mathrm{b}}$ Key Laboratory of Photochemical Conversion and Optoelectronic Materials, Technical Institute of Physics and Chemistry, Chinese Academy of Sciences, Beijing, 100190, \\ China \\ ${ }^{\mathrm{c}}$ State Key Laboratory of High Temperature Gas Dynamics, Institute of Mechanics, Chinese Academy of Sciences, Beijing, 100190, China
}

\section{A R T I C L E I N F O}

\section{Keywords:}

Pressure sensitive paint (PSP)

Bi-layer structured coating

Fast-response

Self-referenced measurement

\begin{abstract}
A B S T R A C T
Herein we report on a biluminophore fast-responding pressure sensitive coating with a bi-layer structure prepared by a non-sensitive reference base paint and subsequently a classic polymer-ceramic pressure sensitive paint (PSP). The coating procedure is fully sprayable and allows the separation of the two luminophores in each layer to prevent their interference that may affect the pressure responsibility. The major advance is the use of solgel process of tetraethoxysilane (TEOS) and methyltriethoxysilane (MTES) to make the bottom reference layer which ensures the spreading and affinity of the top PSP, composed of a room-temperature vulcanizing silicone rubber (RTV), so that very thin PSP layer, i.e. less than $5 \mu \mathrm{m}$, was achieved. Besides, silane-functionalized carbon dots (CDs) was selected as the reference probe, for it could be well-incorporated with the TEOS/MTES matrix, and gained high loading level and hence strong luminescence of the basecoat to overcome the attenuation effect of the top PSP layer. The resultant biluminophore bi-layer fast-responding PSP (denoted as Fast bi-PSP) showed two completed separated emissions with $\lambda_{\max }$ at 430 and $600 \mathrm{~nm}$ for the incorporated CDs and the oxygen sensitive ruthenium complex dye, which enables the utility of intensity ratio from blue and red luminescence for the measurement of surface pressure by following Stern-Volmer relationship. Using a shock tube, the response time of the Fast bi-PSP coating was determined to be $0.4-0.6 \mathrm{~ms}$ according to the chemical composition. The ability of the coating for monitoring the dynamic evolution of full-field surface pressure was demonstrated on sample plates at attack angles of $0^{\circ}$ and $5^{\circ}$. Our work suggests a facile but efficient way for the future design of self-referenced photoluminescent non-contact surface pressure sensing systems.
\end{abstract}

\section{Introduction}

Fast-responding pressure sensitive coating, generated from pressure sensitive paint (PSP), has been studied for two decades for the optical measurement of rapid evolution of global surface-pressure distribution, which is essential to unsteady aerodynamic experiments [1,2]. Basically the pressure sensitivity of PSP is based on an oxygen-quenching mechanism of fluorescence/phosphorescence emission of luminophores trapped in a coating matrix, and therefore the dynamic response of PSP mainly relies on the kinetics of diffusion of environmental oxygen molecules to meet the luminophores in the PSP coating [3,4]. To date, therefore two main types of fast-responding PSPs (Fast PSP), that are, anodized-aluminum (AA) PSP [5,6] and polymer-ceramic (PC) PSP
$[7,8]$, both of which have a porous structure in order to decrease the distance between the oxygen and the luminophore. Compared to AAPSP, classic PC-PSP consists of photoluminescent dyes, ceramic particles and polymer binder dispersed in solvents, which makes it sprayable to form a thin composite film on a model surface [9]. Since the application is more convenient, PC-PSP can be used on various substrates. So far efforts are kept paying to improve the response time. To date, the responsibility of PC-PSPs has reached $10 \mu$ s to microsecond scale $[8,10]$.

Although fast PSP is promising, during the measurement in aerodynamic applications, there are common error sources such as the model movement/deformation between the test and reference conditions, which causes the change of fluorescence in the photo image and

\footnotetext{
*Corresponding author.

*** Corresponding author.

**** Corresponding author.

E-mail addresses: wangqiu@imech.ac.cn (Q. Wang), zhengxie@mail.ipc.ac.cn (Z. Xie), quxz@iccas.ac.cn (X. Qu).
} 
hence affects the dependency on the oxygen concentration driven intensity. To overcome this, the image registration by mapping the windon image onto the wind-off reference image for one-to-one pixel correspondence is thought to be a simple way, but sometime is not so efficient especially if the model moves through a nonuniform illumination field or along the axis [2]. Alternatively, the correction could be made by using a self-referenced two-color regime, a coating containing two or more luminophores, for measuring the emission ratio of two intensity values, in which a second color is produced by a pressureinsensitive reference probe. Based on this strategy, biluminophore PSP (bi-PSP) has been studied by many researchers, not only for solving the problem caused by model movement, but also for the temperature compensation [11,12]. However, it is noted that the use of dual-luminophore system to fast PSP is rare. For a bi-PSP, the dispersion of the two luminophores in the coating is critical to prevent the interference of neighboring dyes which would result in emission quenching, energy transfer, and other unpredictable effects [13]. And to get a fast response, the control the coating microstructure becomes difficult since the phase diagram of the paint is much more complicated, owning to the involvement of liquid-solid interface between the ceramic particles and the dispersing phase. Moreover, fast PSP requires thinner film thickness, and hence faster solvent evaporation. Thus, slight difference on the solubility of the two luminophores will resulted in either aggregation or close packing of the luminophore molecules, leading to quenching, formation of excimer, and/or Förster resonance energy transfer, which will seriously reduce the pressure sensitivity. So far, only a few fast-responding dual-luminophore PC-PSPs were developed, basically with the methodologies of dipping or spraying the dye solution to a pre-prepared ceramic particle containing basecoat [14-16], besides in more recent years, new techniques such as ink-printing and grid-pattern have been applied [17,18].

In this work, we suggested a facile route to get fully sprayable biluminophore PC-PSP with fast response time (denoted as Fast bi-PSP). The coating was constructed with a dual-layer structure, i.e. a substrate coated by a bottom reference layer and subsequently a top pressure responsive layer, which would eliminate the interactions between the luminophores. Theoretically, a coating with a bi-layer architecture is more easily regulated, such as for the fluorescence characteristics, the type of probe, color of emission and intensity of each layer, which can be tuned individually. In particular, classic PSP can be directly utilized as the top layer for favorable oxygen quenching. There are already a few examples of dual-layer PSPs for simultaneous temperature and pressure measurement $[19,20]$. However, problems remain on fast dual-layer PSP, including the possible spectral interference of the dyes, and more important, the reduction of signal strength of the bottom layer because it can be attenuated by top the layer [18], especially if the top PSP contains ceramic particles. To overcome the problems, unique designs are necessary. First, in our regime, silica/polysiloxane was selected to form the bottom matrix. Considering that silicone and polysiloxane are the commonly used as polymer binders of PSP due to the high oxygen permeability [21], we expected that the organosilicone would have ideal wettability and then form strong affinity on the silica bottom layer. This will allow us to obtain very thin PSP on the top, and therefore diminish the shielding effect. Second, we used silane-functionalized carbon dots (CDs) as the reference probe. The silane-functionalization made CDs well-incorporating with the silica matrix. And thus the concentration of CDs in the basecoat could be extremely high, so that it ensures strong emission to further compensate the shadowing of the top PSP. Besides, the CDs were synthesized with blue-emitting, which is well-separated from the red emission of typical oxygen sensitive probes, e.g. tris(4,7-diphenyl-1,10-phenanthroline) ruthenium(II) dichloride $\left(\mathrm{Ru}(\mathrm{dpp})_{3}\right)$ as used in this work, and therefore simplified the data analysis. Images can be recorded just by using a color camera. Herein, we demonstrate the preparation, fluorescence property, responsibility and 2D pressure imaging of the Fast bi-PSP coating.

\section{Experimental section}

\subsection{Materials}

Tis(4,7-diphenyl-1,10-phenanthroline) ruthenium(II) dichloride (Ru $(\mathrm{dpp})_{3}$ ) and room-temperature vulcanizing silicone rubber (RTV) were obtained from Alfa Aesar Chemical Co. Ltd (Shanghai, China) and Momentive/GE Toshiba (New York, USA) respectively. Tetraethoxysilane (TEOS) and methyltriethoxysilane (MTES) were purchased from Macklin Biochemical Co. Ltd (Shanghai, China). $N$-( $\beta$ aminoethyl)- $\gamma$-aminopropylmethylbimethoxy silane (AEAPMS) was bought from Xiangqian Chemical Co., Ltd (Nanjing, China). Titanium silicon oxide $\left(\mathrm{TiSiO}_{4}\right)$ particles were bought from Aladdin Bio-Chem Technology Co. LTD (Shanghai, China). Organic solvents and other compounds were all obtained from Beijing Chemical Reagents Company (China), and were used as received.

\subsection{Preparation of silane-functionalized carbon dots}

Silane-functionalized carbon dots (CDs) was synthesized according to our previous work [22]. Briefly, $10 \mathrm{~mL}$ of organosilane, i.e. AEAPMS, was placed in a $100 \mathrm{~mL}$ three-necked flask, and degassed with argon for $15 \mathrm{~min}$, and was heated to $240^{\circ} \mathrm{C}$. To this $0.5 \mathrm{~g}$ of anhydrous citric acid was added rapidly under vigorous stirring, followed by keeping under the temperature for a few minutes. The final products were then purified by precipitating with petroleum ether for three times.

\subsection{Preparation of biluminophore bi-layer pressure sensitive coating}

The base reference paint was prepared by mixing desired amount of CDs, MTES and TEOS in a mixed solvent of methanol and water $(4: 1 \mathrm{v} /$ v). And volume ratio of the silica precursors to the solvent was 2:5. Then a few drops of acetic acid was added to the dispersion to adjust $\mathrm{pH}$ to 5.0. The base paint was then sprayed onto either glass plates or aluminum plates using a spray gun (F-3, Sheng-Tian, China) with a nozzle diameter of $0.8 \mathrm{~mm}$. The plates were dried in an oven at $80^{\circ} \mathrm{C}$ for $24 \mathrm{~h}$. To prepared the PSP, required amount of $\mathrm{Ru}(\mathrm{dpp})_{3}$, RTV and $\mathrm{TiSiO}_{4}$ particles were mixed in dichloromethane and chlorobenzene at a ratio of $1: 1(\mathrm{v} / \mathrm{v})$, and the mixture was further dispersed by ultrasonication (KH-250DE, Kunshan Hechuang, China). The obtained PSP was sprayed onto the pre-prepared basecoat using the same apparatus. The bi-layer coatings were dried under ambient condition for $12 \mathrm{~h}$ with light avoidance.

\subsection{Characterizations}

\subsubsection{Scanning electron microscopy (SEM)}

Surface and section morphology of the Fast bi-PSP coatings was observed using a scanning electron microscopy (SEM, Hitachi S-4800, Japan) operating at $5 \mathrm{kV}$. For observing the section, samples sprayed on silicon wafer were immersed in liquid nitrogen and then fractured. The samples and sections were sprayed with gold before the observation.

\subsubsection{Fluorescence spectrophotometery}

Sample plate was cut into a size of $13 \times 27 \mathrm{~mm}$, and was placed in a quartz cuvette, sealed with parafilm. The fluorescence spectrum was recorded using a FL970 fluorescence spectrophotometer (Techcomp, China) at room temperature $\left(25^{\circ} \mathrm{C}\right)$ in reflection mode with excitation and emission slits set at 2.5 and $2.5 \mathrm{~nm}$ respectively. For testing the oxygen sensitivity, mixed gas of nitrogen and oxygen in different fraction was purged into the quartz cuvette for at least $3 \mathrm{~min}$. And for checking the photostability, the time scanning mode was used. The samples were continuously irradiated at an excitation of $365 \mathrm{~nm}$, and the emissions at 430 and $600 \mathrm{~nm}$ were recorded respectively for CDs and $\mathrm{Ru}(\mathrm{dpp})_{3}$.

The oxygen sensitivity of the coatings was evaluated using Stern- 
Volmer equation [23],

$\frac{I_{0}}{I}=1+K_{q} \tau_{0}\left[O_{2}\right]$

where $I_{0}$ and $\tau_{0}$ are the fluorescence intensity and excited lifetime in absence of oxygen, herein under $100 \%$ of nitrogen. I is the luminescence intensity at a given oxygen concentration $\left[\mathrm{O}_{2}\right] . \mathrm{K}_{\mathrm{q}} \tau_{0}$ is equal to the Stern-Volmer quenching constant $\left(\mathrm{K}_{\mathrm{sv}}\right)$ in which $\mathrm{K}_{\mathrm{q}}$ is the bimolecular quenching constant. According to Henry's law and Smoluchowski diffusion equation and a suppose of that air contains $21 \%$ of oxygen under $1 \mathrm{~atm}$, the sensitivity of $\mathrm{Ru}(\mathrm{dpp})_{3}$ in the coatings was defined as $\mathrm{I}_{0} / \mathrm{I}_{\text {air }}-1$ or $21 \times \mathrm{K}_{\mathrm{sv}}$ with a unit of $\% / \mathrm{kPa}$.

\subsubsection{Static calibration}

The static calibrations were performed in a custom-built calibration chamber at $25^{\circ} \mathrm{C}$ with a pressure range from 20 to $101 \mathrm{kPa}$, i.e. $0.2-1.0 \mathrm{~atm}$. The low pressure was realized by vacuum pump. The coating in the chamber was excited by a LED lamp at $365 \mathrm{~nm}$ (SJUV 4D104, SJMAEA, China) and the luminescence emitted from the coating was captured by a CCD camera (EOS 700D, Canon, Japan) through a $58 \mathrm{~mm}$ lens (18-55 mm, Canon, Japan) with a long wavepass filter of $410 \mathrm{~nm}$ (LP410, Geng Xu, China). The images were recorded at each given pressure after being held for $3 \mathrm{~min}$. The images were then analyzed using Image $\mathbf{J}$ and Image-Pro Plus 6.0 software for dividing and comparing the intensity in the blue, green and red channels following the software's introductions.

\subsubsection{Dynamic performances of fast bi-PSP coating}

The dynamic performances of the Fast bi-PSP coatings in unsteady aerodynamic test was conducted using a shock tube, as illustrated in Scheme 1 . The shock tube has a square section with inner side length of $84 \times 84 \mathrm{~mm}$, a $0.5 \mathrm{~m}$ long driver section and a $2.2 \mathrm{~m}$ long low pressure section. At $0.8 \mathrm{~m}$ to the end of the low pressure section, a quartz window with a diameter of $60 \mathrm{~mm}$ is placed on the vertical wall of the tube. On the opposite side wall, a groove with a size of $53 \times 30 \times 1.3 \mathrm{~mm}$ was cut for mounting the sample plate $(50 \times 25 \times 1.2 \mathrm{~mm})$. Piezoelectric pressure sensors were equipped on the top side to measure the pressure and incident shock speed. The low pressure shock tube was filled with air at an initial pressure of $50 \mathrm{kPa}$ and the experiments were carried out at room temperature. The Mach number of the incident shock is 1.53 in the tests. In front of the quartz window, a LED array $\left(\lambda_{\text {ex }}=365 \mathrm{~nm}\right)$ and a high-speed CCD camera (FASTCAM Mini AX 200, Japan) with a $410 \mathrm{~nm}$ filter were placed. The camera was connected to a trigger (DG535, USA) and set at a recording speed of $5000 \mathrm{~Hz}(0.2 \mathrm{~ms}$ per picture) with a resolution of $1024 \times 1024$ pixels. The captured images were also analyzed using Image $J$ and Image-Pro Plus 6.0 as mentioned above.

\section{Results and discussion}

\subsection{Preparation of bi-layer biluminophore PSP coating}

The structure of the bi-layer biluminophore fast PSP (Fast bi-PSP) coating is illustrated in Scheme 2, consisted of a bottom reference layer and a top PC-PSP layer. The top PC-PSP layer is classic, with typical $\mathrm{TiSiO}_{4}$ particles incorporated by the room-temperature vulcanizing silicone rubber (RTV), which has ideal transparency to light [24]. The RTV@ $\mathrm{TiSiO}_{4}$ mixture could provide efficient oxygen permeation as well as short distance for the oxygen molecules to meet the dye molecules, because the particles can be uniformly dispersed in RTV matrix within a suitable range of additions. Fast responding PSPs with such a structure haves been well-documented $[3,25,26]$. Herein, we selected Ru(dpp) 3 , instead of Pt(II) meso-tetra(pentafluorophenyl) porphine (PtTFPP), as the oxygen sensitive probe because the former has faster luminescence lifetime [10].

The bottom reference layer of the Fast bi-PSP coating was made through sol-gel process of tetraethoxysilane (TEOS) together with methyltriethoxysilane (MTES) to embed silane-functionalized CDs. CDs have been widely applied in imaging devices and nano-sensing systems because of the high photostability, high quantum yield, tunable luminescent color [22,27-30]. Besides, CDs can be easily functionalized to benefit the dispersion in various materials. Like in this work, the functionalization with surface methylsilane groups endowed homogeneous immobilization of CDs in the silica matrix due to the formation of $\mathrm{Si}-\mathrm{O}$ bonds between CDs and the TEOS/MTES precursors [31,32]. Moreover, the TEOS/MTES regime provided extra features. For example, it is sprayable. And the wettability of the formed basecoat can be tuned by simply changing the TEOS/MTES ratio for a favorable spreading of PSP on its surface. Besides, the inclusion of MTES to TEOS helped to get crack-free thin films [33]. Furthermore, since octamethyl cyclotetrasiloxane is a main component of RTV for the formation of silicone via a ring opening process, the residue hydroxyl groups on the TEOS/MTES hybrid film surface can also bond to the silicone to stabilize the interface and thus offers high affinity between the reference layer and the sensing layer. Herein, an optimized TEOS/MTES ratio of $3: 7(\mathrm{v} / \mathrm{v})$ was used. All the features resulted in the generation of very thin PC-PSP layer for the fast pressure response.

From the SEM images, the bi-layer structure can be clearly seen on a silicon substrate (Fig. 1a). The thickness of the TEOS/MTES layer is ca. $3 \mu \mathrm{m}$ and the PSP layer is ca. $2.5-4 \mu \mathrm{m}$, controlled by the spraying process. From EDX observation, the distribution of titanium element confirms the interface between TEOS/MTES and PC-PSP layers (Fig. 1b), which proves that the TEOS/MTES layer is an eligible basecoat to PC-PSP. From the optical images, one can see the uniform coatings gained on glass substrate by spray technique (Fig. 1c). The construction of a bi-layer structure is a general strategy to separate two kinds of probes and avoid the possible interaction between the dye molecules. The method is facile for achieving dual-luminophore sensing

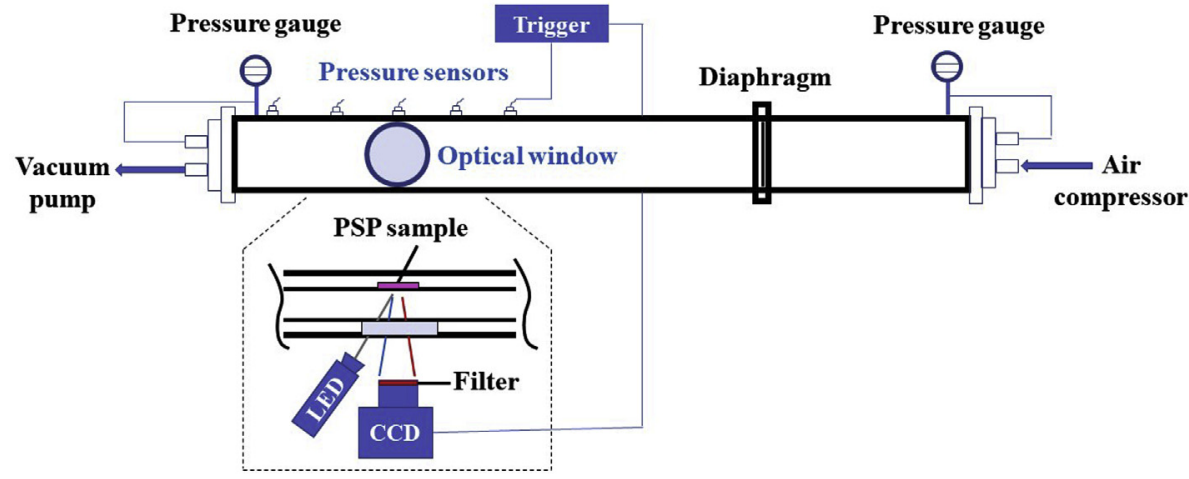

Scheme 1. Illustrative scheme of the shock tube for testing the unsteady aerodynamic performance of PSP coating. 


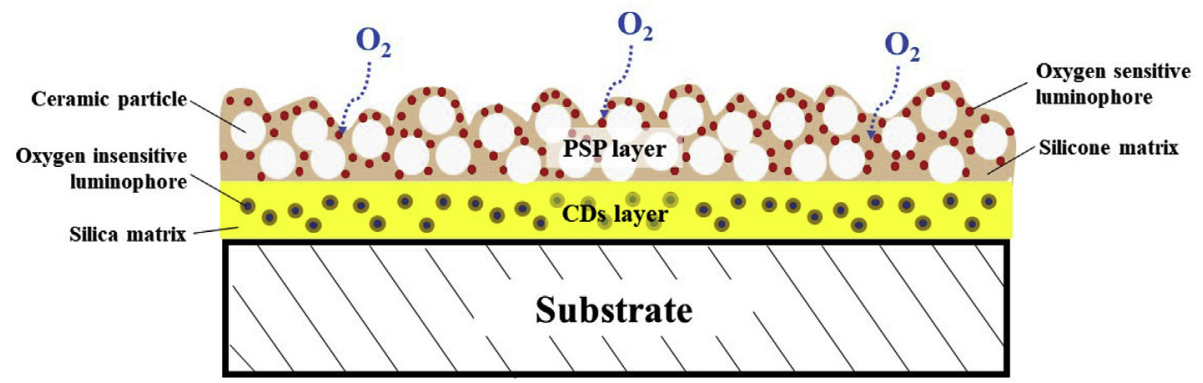

Scheme 2. Structure of Fast bi-PSP coating. The oxygen insensitive and the oxygen sensitive luminophores in this work are CDs and Ru(dpp) ${ }_{3}$, respectively.

(a)
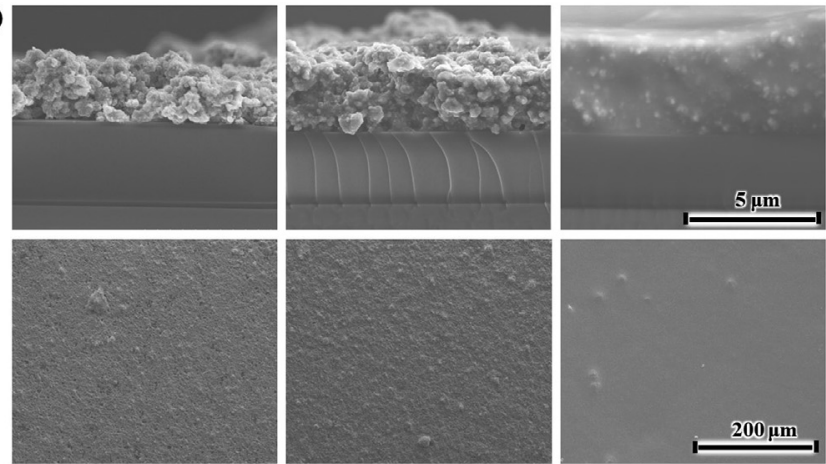

(b)
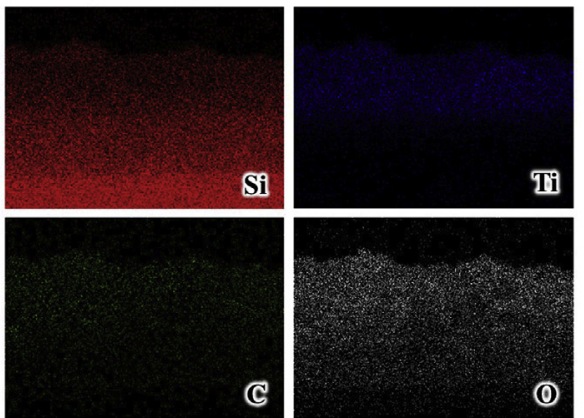

(c)
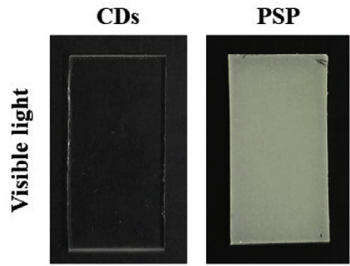

Bi-PSP
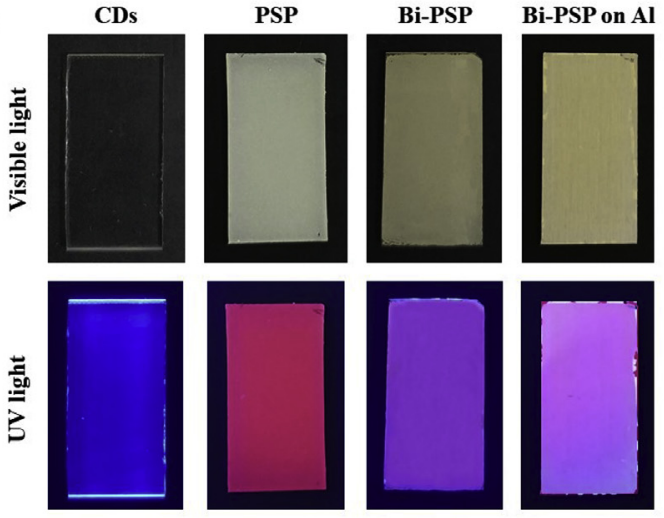

Fig. 1. (a) SEM images of the section (up) and the top view (down) of Fast biPSP coatings containing $10 \mathrm{wt} \%$ of CDs, $0.6 \mathrm{mg} / \mathrm{m}^{2}$ of $\mathrm{Ru}(\mathrm{dpp})_{3}, 0.6 \mathrm{~g} / \mathrm{m}^{2}$ of $\mathrm{TiSiO}_{4}$ and $0.4,0.8$ and $2.0 \mathrm{~g} / \mathrm{m}^{2}$ of RTV (from left to right). (b) Elemental maps (EDX) of the Fast bi-PSP coating. (c) Optical pictures of single layer CDs/TEOS/ MTES coating, PC-PSP coating and bi-layer Fast bi-PSP coating on glass substrate, and Fast bi-PSP coating on aluminum substrate under visible light and UV irradiation.

system. In addition to glass and silicon substrates, Fast bi-PSP on different substrates, e.g. aluminum plate, can be also prepared, as shown in Fig. 1c. (a)
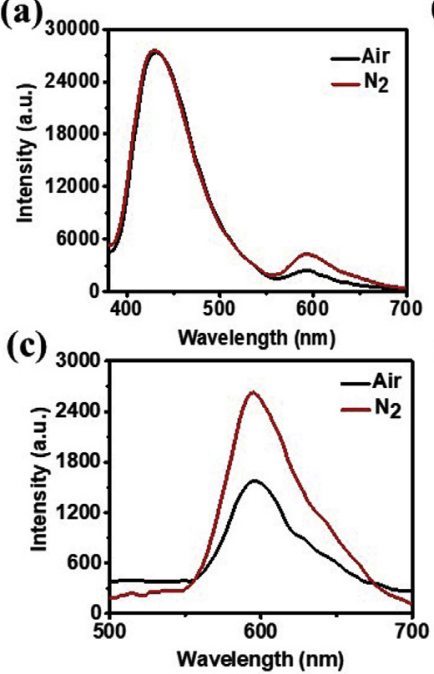

(b)

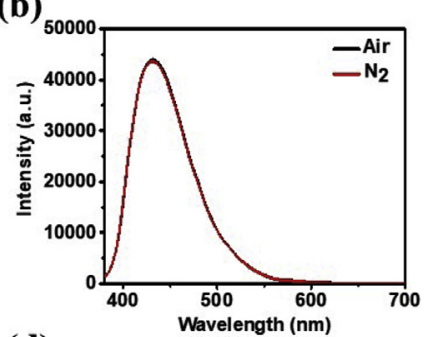

(d)

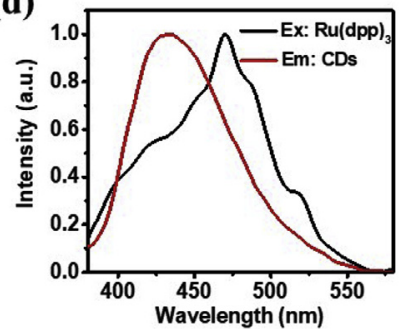

Fig. 2. Reflection emission spectra of Fast bi-PSP (a) and CDs/TEOS/MTES single layer coating (b) and single layer PSP (c) with $\lambda_{\text {ex }}$ of $365 \mathrm{~nm}$. The coatings contain $10 \mathrm{wt} \%$ of CDs, $1.2 \mathrm{mg} / \mathrm{m}^{2}$ of $\mathrm{Ru}(\mathrm{dpp})_{3}, 0.6 \mathrm{~g} / \mathrm{m}^{2}$ of $\mathrm{TiSiO}_{4}$ and $2.0 \mathrm{~g} / \mathrm{m}^{2}$ of RTV where the components exist. And overlap of the emission spectrum of single layer CDs coating $\left(\lambda_{\text {ex }}=365 \mathrm{~nm}\right)$ and the excitation spectrum of single layer PSP coating $\left(\lambda_{\mathrm{em}}=600 \mathrm{~nm}\right)(\mathrm{d})$.

\subsection{Fluorescence property of Fast bi-PSP}

The fluorescence property of the Fast bi-PSP coatings was first evaluated using fluorescence spectrophotometer. As shown in Fig. 2a, the Fast bi-PSP coating exhibits two emission peaks centered at $430 \mathrm{~nm}$ and $600 \mathrm{~nm}$, when excited at $365 \mathrm{~nm}$, representing to the fluorescence of $\mathrm{CDs}$ and $\mathrm{Ru}(\mathrm{dpp})_{3}$ respectively. The fluorescence intensity of each band can be adjusted individually by changing the composition of the luminophores in the corresponding layers. Nevertheless, for a double layer structure, a major concern is the influence of the PSP top layer on the emission of the CDs basecoat. It first noticed from Fig. 1c that the mixed color of the excited blue-emitting CDs and red-emitting $\mathrm{Ru}(\mathrm{dpp})_{3}$ infers acceptable attenuation of the cover coating, i.e. PC-PSP, to the underlying CDs, attributed to the low thickness of the PSP top layer. Fluorescence spectra further show that the center position of both CDs and $\mathrm{Ru}(\mathrm{dpp})_{3}$ emission $\left(\lambda_{\max }\right)$ of the Fast bi-PSP coating does not change when compared to the neat CDs or $\mathrm{Ru}(\mathrm{dpp})_{3}$ single layer coating, despite they were examined under nitrogen or in air, implying that the coupling of $\mathrm{CDs}$ and $\mathrm{Ru}(\mathrm{dpp})_{3}$ was not happen. On the other hand, the fluorescence strength of CDs in the Fast bi-PSP coating indeed becomes weaker than that of neat CDs coating with similar composition (Fig. 2b), because of the inevitable shielding effect of the top PSP layer. Nonetheless, since the CDs have high quantum yields, with suitable concentration, e.g. 3-25 wt\%, the bottom layer can still exhibit high emission intensity even covered by the PSP top layer. Meanwhile, we also notice that the intensity of $\mathrm{Ru}(\mathrm{dpp})_{3}$ emission increased 
(a)
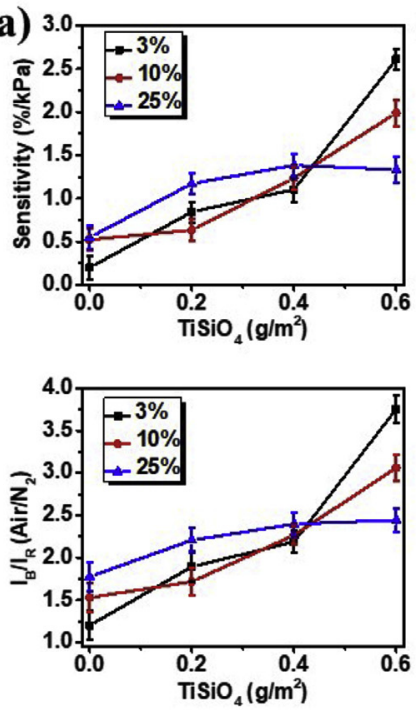

(d)
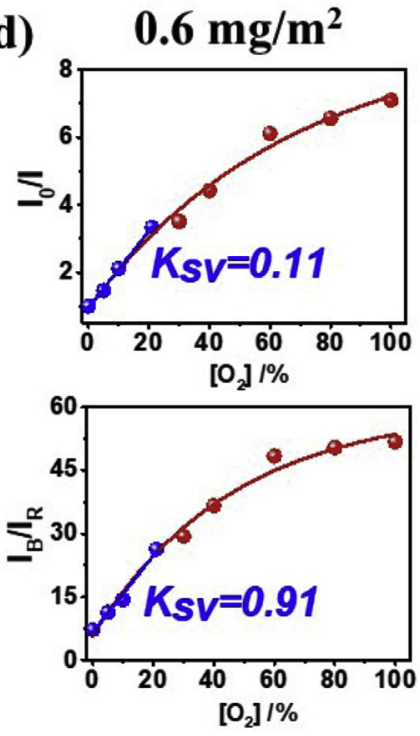

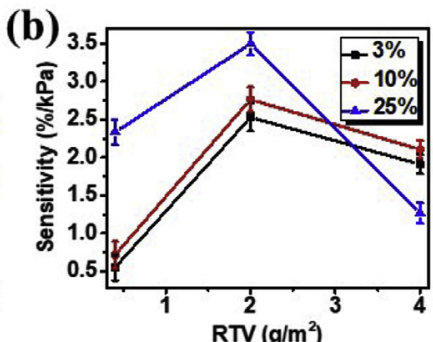

(c)
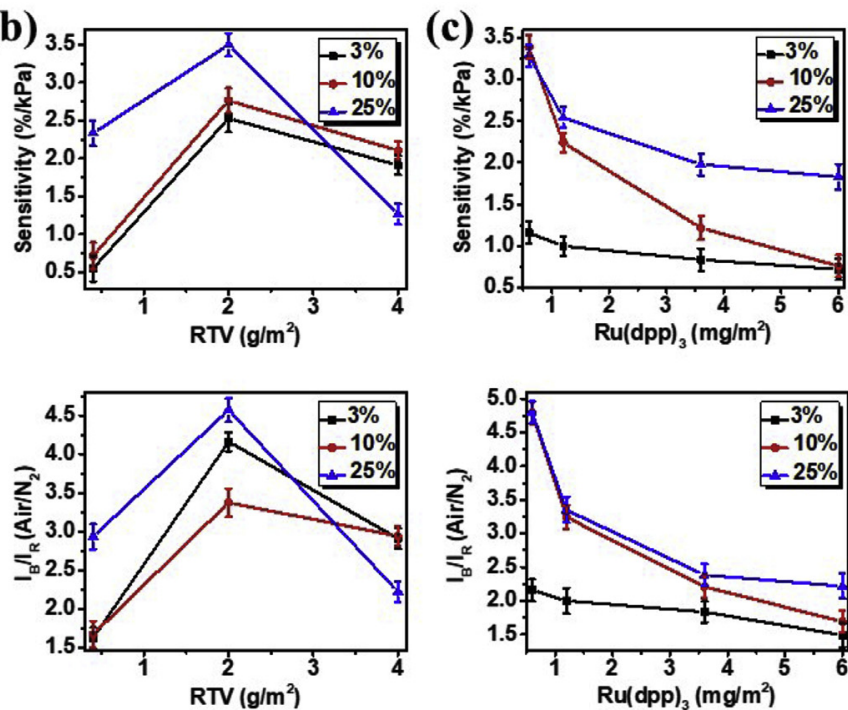

Fig. 3. Effect of chemical composition on the sensitivity of the Fast bi-PSP coatings. Top row, sensitivity of $\mathrm{Ru}$ $(\mathrm{dpp})_{3}$. Bottom row, intensity ratio of emission at $430 \mathrm{~nm}-600 \mathrm{~nm}$, i.e. $\mathrm{I}_{\mathrm{B}} / \mathrm{I}_{\mathrm{R}}$. (a) Varying $\mathrm{TiSiO}_{4}$ content with RTV and $\mathrm{Ru}(\mathrm{dpp})_{3}$ keeping at $2.0 \mathrm{~g} / \mathrm{m}^{2}$ and $1.2 \mathrm{mg} / \mathrm{m}^{2}$. (b) Varying RTV amount with $\mathrm{TiSiO}_{4}$ and $\mathrm{Ru}(\mathrm{dpp})_{3}$ keeping at $0.6 \mathrm{~g} / \mathrm{m}^{2}$ and $1.2 \mathrm{mg} / \mathrm{m}^{2}$; (c) Varying $\mathrm{Ru}(\mathrm{dpp})_{3}$ addition with $\mathrm{TiSiO}_{4}$ and RTV keeping at $0.6 \mathrm{~g} / \mathrm{m}^{2}$ and $2.0 \mathrm{~g} / \mathrm{m}^{2}$. The $\%$ in each figure represents the weight percentage of silane-functionalized CDs in the silica layer. (d) The $\mathrm{I}_{0} / \mathrm{I}$ of $\mathrm{Ru}$ $(\mathrm{dpp})_{3}$ and $\mathrm{I}_{\mathrm{B}} / \mathrm{I}_{\mathrm{R}}$ of the Fast bi-PSP coatings containing $10 \mathrm{wt} \%$ of $\mathrm{CDs}$, $0.6 \mathrm{~g} / \mathrm{m}^{2}$ of $\mathrm{TiSiO}_{4}$ and $2.0 \mathrm{~g} / \mathrm{m}^{2}$ of RTV, but with varying concentration of $\mathrm{Ru}(\mathrm{dpp})_{3}$ as a function of oxygen fraction. $\mathrm{K}_{\mathrm{sv}}$ is the slope in the linear fit region. The excitation wavelength for (a-d) was $365 \mathrm{~nm}$.
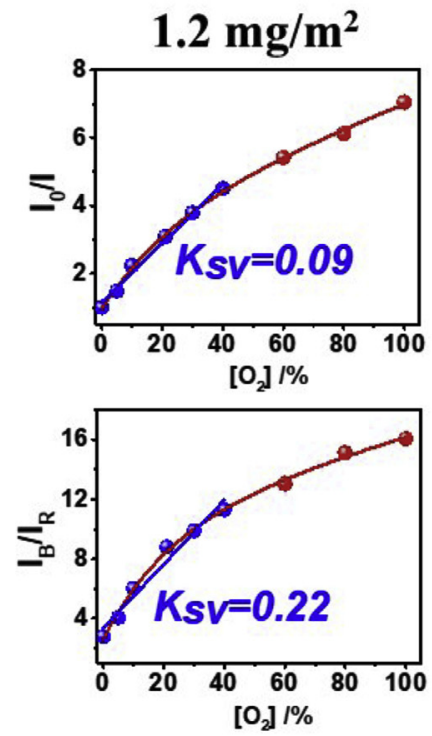

significantly in Fast bi-PSP coating in comparison with the neat Ru $(\mathrm{dpp})_{3}$ coating (Fig. 2c). The phenomenon suggests another possibility for the decrease of CDs fluorescence in Fast bi-PSP, that is, an energy transfer mechanism from $\mathrm{CDs}$ to $\mathrm{Ru}(\mathrm{dpp})_{3}$. And the hypothesis is supported by the overlap of CDs emission and $\mathrm{Ru}(\mathrm{dpp})_{3}$ excitation spectra (Fig. 2d). From Fig. 2, it is important to reveal that varying atmosphere between nitrogen and air only changes the intensity of $\mathrm{Ru}(\mathrm{dpp})_{3}$ in the Fast bi-PSP coating, whilst the emission strength of CDs peak keeps unchanged. Considering the well-separated emission peaks of CDs and $\mathrm{Ru}(\mathrm{dpp})_{3}$, it confirms that we enable to measure the surface oxygen concentration or fraction through not only the fluorescence intensity of $\mathrm{Ru}(\mathrm{dpp})_{3}$, but also via the intensity ratio of $\mathrm{CDs}$ and $\mathrm{Ru}(\mathrm{dpp})_{3}$, i.e. $\mathrm{I}_{430}$ / $\mathrm{I}_{600}$ or $\mathrm{I}_{\mathrm{B}} / \mathrm{I}_{\mathrm{R}}$.

\subsection{Oxygen sensitivity of Fast bi-PSP}

Oxygen sensitivity is a key parameter to evaluate the performance of PSP, which is instinctively determined by the sensitive probe. However for a PC-PSP, the oxygen sensitivity is also influenced by the composition of $\mathrm{TiSiO}_{4}$ particle and RTV. In the current regime, Fig. 3a shows that increasing $\mathrm{TiSiO}_{4}$ amount causes the increase of the
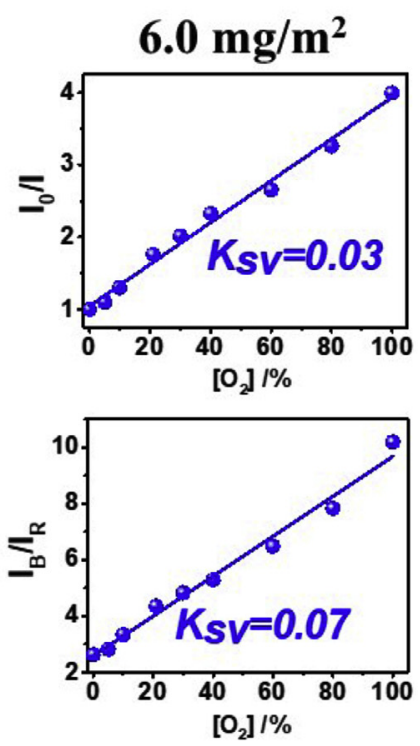

apparent sensitivity of $\mathrm{Ru}(\mathrm{dpp})_{3}$ in the PSP coatings. The result is reasonable because the blending of inorganic particles into the silicone matrix shortens the distance between the oxygen molecules to $\mathrm{Ru}$ (dpp) ${ }_{3}$, and thus increases the possibility for the oxygen molecules to meet the probe molecules and hence improves the quenching efficiency. However, the influence of RTV amount to the oxygen sensitivity of PSP is somehow interesting, since an optimal content of RTV is observed (Fig. 3b). While the reduction of oxygen sensitivity with more RTV can be explained by the increase of PSP thickness (Fig. 1a), hence with less oxygen molecules permeating into deeper place, surprisingly lower level of RTV also resulted in poorer sensitivity, which could be only ascribed as the distribution of probe in the PSP matrix. Ru(dpp) ${ }_{3}$ enable to homogeneously disperse in the silicone matrix. Nevertheless, lower RTV content will still lead to the distribution of probe molecules to the $\mathrm{TiSiO}_{4}$ surface. Previous studies have proven that the couple of ruthenium photosensor with an inorganic surface generates a confinement effect and therefore changes the photoluminescence property [34,35]. To demonstrate the effect, we changed the concentration of $\mathrm{Ru}(\mathrm{dpp})_{3}$ while the amount of $\mathrm{TiSiO}_{4}$ and RTV kept at the same. Indeed, it reveals a general decrease of sensitivity of the coatings with the increase of $\mathrm{Ru}$ (dpp) ${ }_{3}$ (Fig. 3c). Overall, the highest sensitivity of the tested Ru(dpp) ${ }_{3}$ 
is $>3 \% / \mathrm{kPa}$ in the as-prepared PSP, converted from the oxygen fraction (Fig. 3). Meanwhile, the sensitivity of the Fast bi-PSP was also investigated by checking the intensity ratio of $\mathrm{CDs}$ to $\mathrm{Ru}(\mathrm{dpp})_{3}\left(\mathrm{I}_{\mathrm{B}} / \mathrm{I}_{\mathrm{R}}\right)$ in nitrogen and in air. As expected, its relationship shows similar trends as that to the individual Ru(dpp) ${ }_{3}$ (Fig. 3), owning to the stable fluorescent property of CDs, giving a constant $\mathrm{I}_{B}$ under various oxygen concentration. Therefore, both intensity variation of $\mathrm{Ru}(\mathrm{dpp})_{3}$ emission and $I_{B} / I_{R}$ from the two probes can be used to determine the surface pressure change.

\subsection{Static pressure calibration and photostability}

The Fast bi-PSP coatings, prepared using spraying method, with $10 \mathrm{wt} \%$ concentration of CDs in the base paint, $6.0 \mathrm{mg} / \mathrm{m}^{2}$ of $\mathrm{Ru}(\mathrm{dpp})_{3}$ and $0.6 \mathrm{~g} / \mathrm{m}^{2}$ of $\mathrm{TiSiO}_{4}$ particles in the PSP, were used for the investigation of pressure sensitivity. Although according to Fig. 3c, PSP containing $6 \mathrm{mg} / \mathrm{m}^{2}$ of $\mathrm{Ru}(\mathrm{dpp})_{3}$ may not have the highest oxygen sensitivity, relatively higher amount of probe will give higher fluorescence intensity so that it will benefit the $2 \mathrm{D}$ imaging. Moreover, a calibration on intensity variation of $\mathrm{Ru}(\mathrm{dpp})_{3}$ or $\mathrm{I}_{\mathrm{B}} / \mathrm{I}_{\mathrm{R}}$ as a function of oxygen fraction demonstrates that with more probe molecules, the quenching effect keeps unsaturated at higher oxygen level, providing wider linear-responding region of oxygen fraction to the fluorescence emission (Fig. 3d).

The static pressure calibration of the Fast bi-PSP coatings with various binder content were tested in a vacuum chamber and the results were plotted in Fig. 4. Compared to the optical images, through an analysis of the intensity ratio of the recorded pictures, i.e. blue channel to red channel $\left(\mathrm{I}_{\mathrm{B}} / \mathrm{I}_{\mathrm{R}}\right)$, one can more obviously observe the pressure change of the bi-PSP (Fig. 4a). Calibration curves shows that all samples have a linear relationship between the pressure and the calculated $I_{B} / I_{R}$ within the pressure range, when RTV content varying from $0.4 \mathrm{~g} / \mathrm{m}^{2}$ to $2.0 \mathrm{~g} / \mathrm{m}^{2}$ (Fig. 4b). According to the slope of the static calibration curves, the effect of RTV content on the sensitivity has the same order as those measured by the fluorescence tests (Fig. 3b). Thus, under the experimental conditions, the Stern-Volmer relation can be inverted to the following equation,

$\frac{I_{B}}{I_{R}}=A^{\prime}+B^{\prime} \frac{p}{p_{0}}$

where $I_{B} / I_{R}$ is the real-time data at a given place in the image and $p_{0}$ is the referenced pressure. The $\mathrm{I}_{\mathrm{B}} / \mathrm{I}_{\mathrm{R}}$ data at each element in the image could be recorded for drawing a 2D pressure map. But for getting a calibration curve, we used the average values of the elements along a line, as shown in Fig. 4a, for better accuracy.

The photostability of the Fast bi-PSP coatings was also investigated and the results are presented in Fig. 5. It is seen that the photodegradation of the PSP, i.e. $\mathrm{Ru}(\mathrm{dpp})_{3}$, is around $14 \%-25 \%$, depending on the level of RTV content (Fig. 5a). Such values is normal for PC-PSP as mentioned in literature $[3,9,10]$, since the decomposition of the dye molecules can be facilitated by the $\mathrm{TiO}_{2}$ composition in the ceramic particles which works as a photocatalyst under the illumination of UV light $[7,36]$. Increasing RTV portion in the PSP layer improves the photostability owning to better distribution of the dye molecules in the binder matrix, rather than packing to particle surface. However, more RTV also means the increase of permeating distance of oxygen molecules to the dyes, which will lead to negative effect on the dynamic responsibility of the PSP. On the other hand, it is recognized that the luminescent intensity of CDs also reduces slightly with time, and as shown in Fig. 5 b, with a decrease of $5 \%-7 \%$ to the original intensity. The value is much lower than that of $\mathrm{Ru}(\mathrm{dpp})_{3}$, because CDs is more stable against UV irradiation [37]. Different from a conventional PSP, in the self-referenced system, the photobleaching of the reference probe will compensate the aging kinetics of the sensitive probe, resulting in less $I_{B} / I_{R}$ ratio change as a function of time (Fig. 5 c).
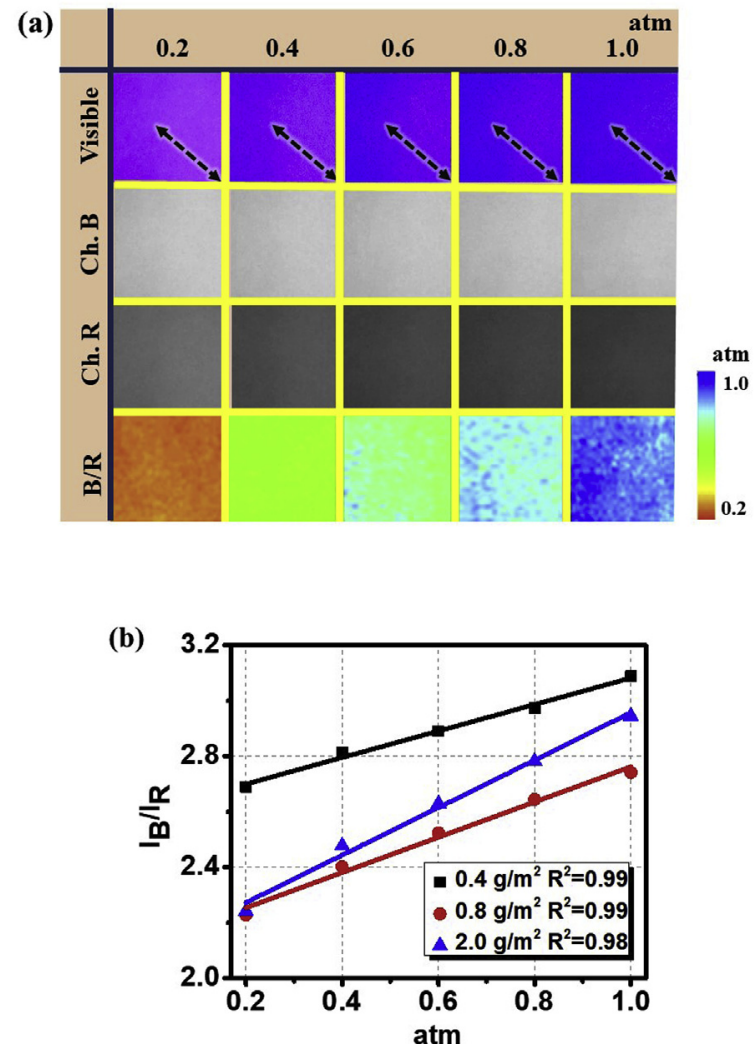

Fig. 4. Static calibration of Fast bi-PSP. (a) 2D images of surface pressure distribution, derived from optical pictures taken under predetermined pressure followed by processing using commercial software to divide the color from RGB to blue, green (not shown) and red channels. (b) Static calibration curves of Fast bi-PSP based on the intensity ratio of blue channel to red channel. The $I_{B} / I_{R}$ value was averaged from the elements along the black dash line as shown in (a). The Fast bi-PSP contains $10 \mathrm{wt} \%$ of CDs, $6.0 \mathrm{mg} / \mathrm{m}^{2}$ of $\mathrm{Ru}(\mathrm{dpp})_{3}, 0.6 \mathrm{~g} / \mathrm{m}^{2}$ of $\mathrm{TiSiO}_{4}$ and $0.4 \mathrm{~g} / \mathrm{m}^{2}$ of RTV for (a) and mentioned amount of RTV in the figure of (b).

\subsection{Response time and $2 D$ pressure imaging}

The sprayed Fast bi-PSP coatings, made from a base paint containing $10 \mathrm{wt} \%$ of CDs, and a PSP containing $6.0 \mathrm{mg} / \mathrm{m}^{2}$ of $\mathrm{Ru}(\mathrm{dpp})_{3}$, $0.6 \mathrm{~g} / \mathrm{m}^{2}$ of $\mathrm{TiSiO}_{4}$ and 0.4 or $0.8 \mathrm{~g} / \mathrm{m}^{2}$ of RTV were used to examine the dynamic responding time in the shock tube (Scheme 1). Higher level RTV was not considered because of the fast-responding aim. It is no doubt that more binder over the ceramic particles will significantly reduce the responsibility of PSP, since the characteristic time $\tau_{\text {diff }}$ is in proportion to the square of binder thickness (h), as described in the following equation [38],

$\tau_{\text {diff }} \propto \frac{h^{2}}{D_{m}}$

where $\mathrm{D}_{\mathrm{m}}$ are the diffusion coefficient of the binder material. In Fig. 1a, one can see that the roughness of the PSP coating surface decreases with the increase of RTV content when $\mathrm{TiSiO}_{4}$ amount was the same, referring to thicker silicone layer on the particles.

The dynamic response results of the tested Fast bi-PSP coatings are shown in Fig. 6, in which it proves that the coatings enable to record the sudden pressure change caused by the incident shock as well as the reflected shock wave in the tube. The response time is 0.4 and $0.6 \mathrm{~ms}$ to the coatings containing 0.4 and $0.8 \mathrm{~g} / \mathrm{m}^{2}$ of RTV in the PSP layer, which once again confirmed the relationship between the binder content and the response time. Besides, the analysis on the images also demonstrates the self-reference ability of the Fast bi-PSP. As shown in Fig. 6, the derived blue and red intensities show that the $I_{B} / I_{R}$ variation is 

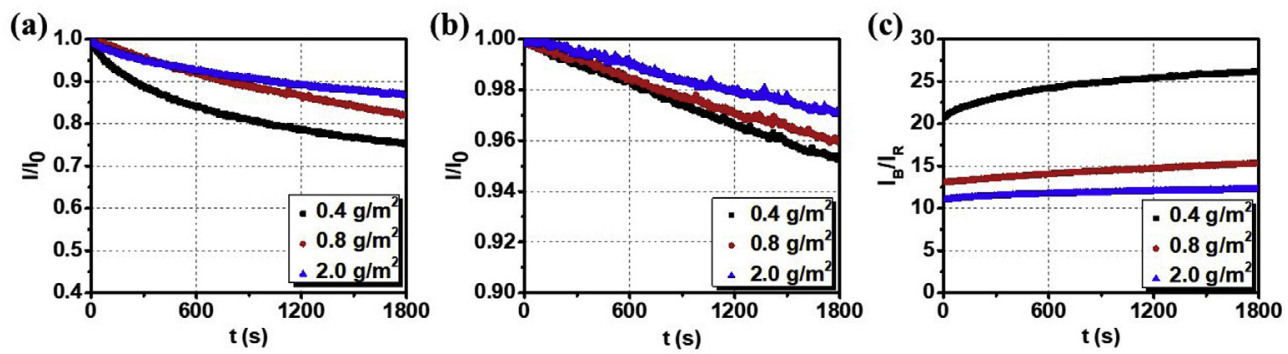

Fig. 5. Photostability of Fast bi-PSP coatings containing $10 \mathrm{wt} \%$ of $\mathrm{CDs}, 6.0 \mathrm{mg} / \mathrm{m}^{2}$ of Ru(dpp $)_{3}, 0.6 \mathrm{~g} / \mathrm{m}^{2}$ of $\mathrm{TiSiO}_{4}$ and various amount of RTV under an irradiation at $365 \mathrm{~nm}$. (a) $\lambda_{\mathrm{em}}=600 \mathrm{~nm}$. (b) $\lambda_{\mathrm{em}}=430 \mathrm{~nm}$. (c) $\mathrm{I}_{430} / \mathrm{I}_{600}$. $\mathrm{I}_{0}$ in this figure is the intensity at the start of the test.

originated from the responsibility of $\mathrm{Ru}(\mathrm{dpp})_{3}$ whereas the luminescence of CDs in the bottom layer was almost unchanged during the aerodynamic process. Compared to the blend-mixing of two luminophores, by separating the reference and sensitive probes in different layers, the properties of the top PSP have no significant difference to a classic single-layer PSP, and hence its properties can be more easily controlled and tuned. In this work the construction of the reference coating was also facile which allows the use of any kind of probe with silane functionalization. And the dual-luminophore PSP is fully sprayable which is convincing for the practical use. To get desired responsibility and optical observation, the stability and morphology control of the top layer is critical. Herein, the silica and silicone binders of the two layers are well-incorporated with each other to get stabilized PSP on top, and such Fast bi-PSP is ideal for unsteady pressure measurement with high spatial resolution in intensity ratio mode.

The 2D imaging of the Fast bi-PSP coatings in shock tube is demonstrated in Fig. 7. Two attack angles, i.e. $0^{\circ}$ and $5^{\circ}$, were tested. With a frame rate of $5000 \mathrm{~Hz}$, the camera have recorded the visible color change on the coating surface (Fig. 7), a definite indication of strong photoluminescence of the coatings. Subsequently, the pressure evolution can be more clearly presented using the ratio images by comparing the blue and red channels, where the sharp change of pressure and pressure distribution are captured picture-by-picture, taken within $0.4 \mathrm{~ms}$ (Fig. 7), which is the response time of the coating. Especially for the test at $5^{\circ}$ attack angle, the $\mathrm{I}_{\mathrm{B}} / \mathrm{I}_{\mathrm{R}}$ map suggests a nonuniform pressure increase from the far-end to near-end of the plate, and later on a heterogeneous pressure distribution on the plate under the incident shock wave. The above demonstration confirms the promising capacity of the Fast bi-PSP coating for aerodynamic testing of surface pressure.

(a)

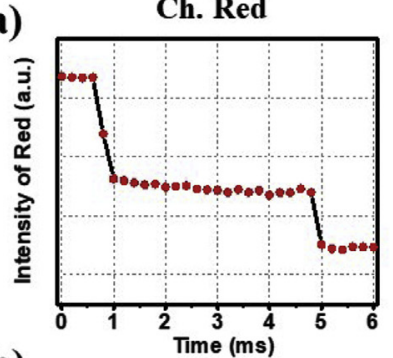

(b)

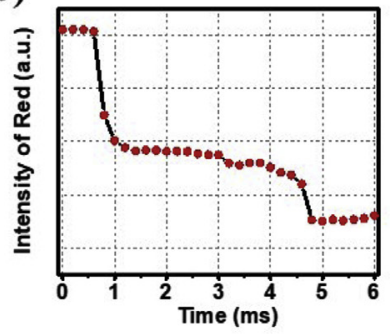

Ch. Blue
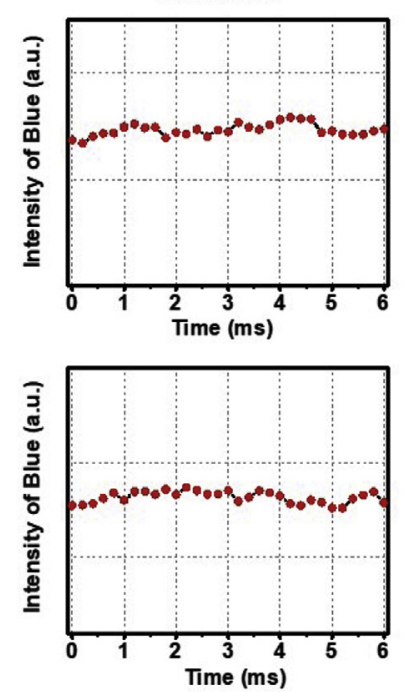

\section{Conclusions}

In this work, we developed a mothed to build biluminophore fastresponding pressure sensitive coatings (Fast bi-PSP coating) by the construction of a double layer structure to disperse the reference probe and the response probe in separate layers in order to avoid potential inter-probe interruption. Both of the reference paint and the PSP are sprayable. And by using silane-functionalized carbon dots, the reference silica coating has strong photoluminescence and meanwhile ideal affinity to the top PSP coating consisted of silicone matrix. This resulted in thin PSP layer and limit attenuation effect to the reference layer. Ratio comparison method on blue and red emissions is established for measuring the surface oxygen fraction and hence the surface pressure. Calibrations confirmed that the pressure value can be calculated by following the Stern-Volmer relation. The response time of the bi-layer coating is $0.4-0.6 \mathrm{~ms}$ to the surface pressure change as determined using a shock tube, which endowed the ability of the Fast bi-PSP to record the $2 \mathrm{D}$ pressure evolution on a model plate in high-speed, unsteady aerodynamic testing. Our results are expected to benefit the future design of self-referenced oxygen, pressure or multiple signal (e.g. pressure and temperature) photoluminescence sensing systems.

\section{Conflicts of interest}

There are no conflicts to declare.

\section{Acknowledgements}

This work is financially supported by Chinese Academy of Sciences (CXJJ-15M060) and National Natural Science Foundation of China
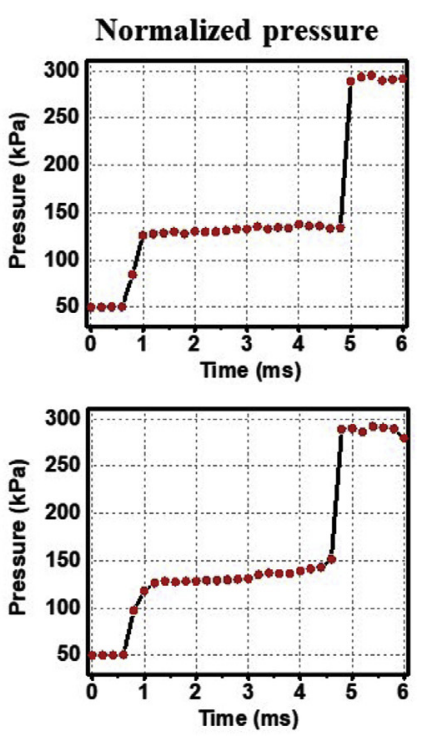

Fig. 6. Response of two Fast bi-PSP coatings to the incident and reflected shock wave in the shock tube. The data were calculated using the software as mentioned above from the captured pictures at a frame rate of $5000 \mathrm{~Hz}$. The Fast bi-PSP contains $10 \mathrm{wt} \%$ of CDs, $6.0 \mathrm{mg} / \mathrm{m}^{2}$ of $\mathrm{Ru}(\mathrm{dpp})_{3}, 0.6 \mathrm{~g} / \mathrm{m}^{2}$ of $\mathrm{TiSiO}_{4}$ and 0.4 (a) and 0.8 (b) $\mathrm{g} / \mathrm{m}^{2}$ of RTV. The excitation wavelength was $365 \mathrm{~nm}$. 

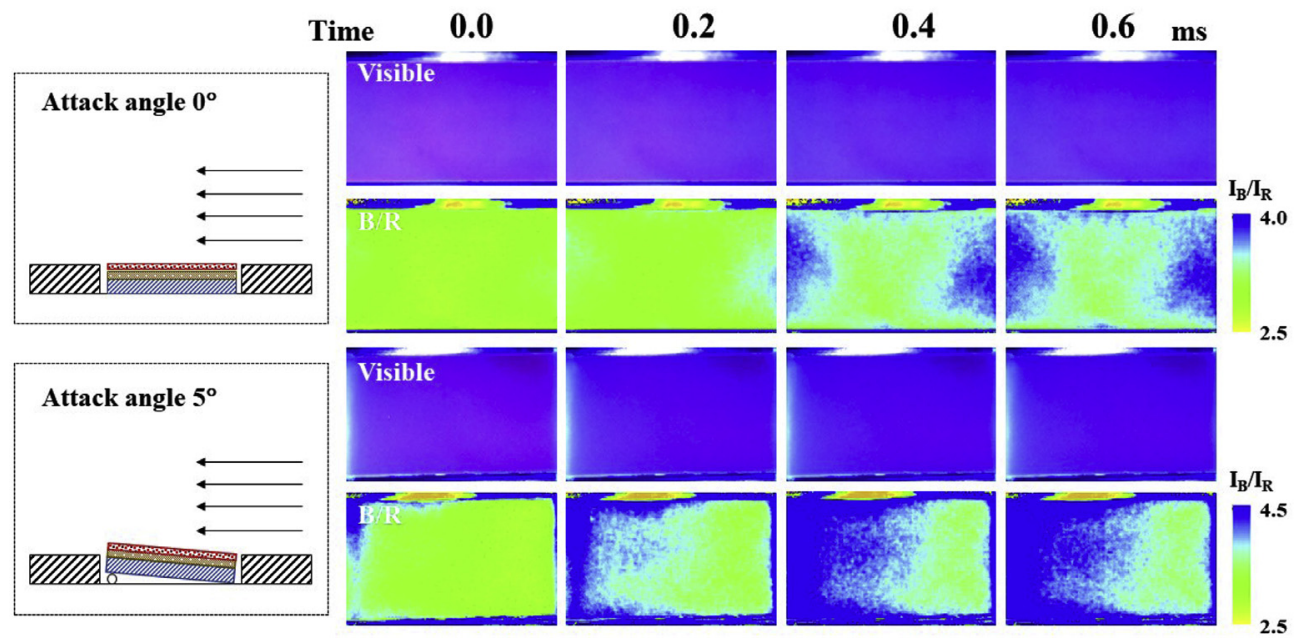

Fig. 7. Visible and processed (blue channel over red channel) images of plate surfaces covered by Fast bi-PSP containing $10 \mathrm{wt} \%$ of $\mathrm{CDs}, 6.0 \mathrm{mg} / \mathrm{m}^{2}$ of Ru(dpp) ${ }_{3}$, $0.6 \mathrm{~g} / \mathrm{m}^{2}$ of $\mathrm{TiSiO}_{4}$ and $0.4 \mathrm{~g} / \mathrm{m}^{2}$ of RTV in response to the shock wave at attack angles of $0^{\circ}$ and $5^{\circ}$. The excitation wavelength was $365 \mathrm{~nm}$.

(21875267, 51561145004). Prof. X. Jin and Prof. L. Chen of Institute of Chemistry, Chinese Academy of Sciences are acknowledged for their valuable advices.

\section{References}

[1] J.H. Bell, E.T. Schairer, L. A H, R.D. Mehta, Surface pressure measurements using luminescent coatings, Annu. Rev. Fluid Mech. 33 (2003) 155-206.

[2] J.W. Gregory, H. Sakaue, T. Liu, J.P. Sullivan, Fast pressure-sensitive paint for flow and acoustic diagnostics, Annu. Rev. Fluid Mech. 46 (2014) 303-330.

[3] Y. Egami, S. Konishi, Y. Sato, Y. Matsuda, Effects of solvents for luminophore on dynamic and static characteristics of sprayable polymer/ceramic pressure-sensitive paint, Sensor. Actuat. A Phys. 286 (2019) 188-194.

[4] Y. Mao, Q. Zhao, J. Wu, T. Pan, B. Zhou, Y. Tian, A highly sensitive and fast-responding oxygen sensor based on POSS-containing hybrid copolymer films, J. Mater. Chem. C 5 (2017) 11395-11402.

[5] H. Sakaue, K. Morita, Y. Iijima, Y. Sakamura, Response time scales of anodizedaluminum pressure-sensitive paints, Sensor. Actuat. A Phys. 199 (2013) 74-79.

[6] H. Sakaue, K. Ishii, Optimization of anodized-aluminum pressure-sensitive paint by controlling luminophore concentration, Sensors 10 (2010) 6836-6847.

[7] A. Pandey, J.W. Gregory, Step response characteristics of polymer/ceramic pressure-sensitive paint, Sensors 15 (2015) 22304-22324.

[8] M. Kameda, H. Seki, T. Makoshi, Y. Amao, K. Nakakita, A fast-response pressure sensor based on a dye-adsorbed silica nanoparticle film, Sensor. Actuator. B Chem. 171-172 (2012) 343-349.

[9] P. Di, G. Feng, Y. Li, Y. Liu, A novel sprayable fast-responding pressure-sensitive paint based on mesoporous silicone dioxide particles, Sensor. Actuat. A Phys. 279 (2018) 390-398.

[10] Y. Egami, Y. Sato, S. Konishi, Development of sprayable pressure-sensitive paint with a response time of less than 10 Hs, AIAA J. 57 (2019) 1-6.

[11] K. Mitsuo, K. Asai, M. Hayasaka, M. Kameda, Temperature correction of PSP measurement using dual-luminophor coating, J. Vis. 6 (2003) 213-223.

[12] G.E. Khalil, C. Costin, J. Crafton, G. Jones, S. Grenoble, M. Gouterman, J.B. Callis, L.R. Dalton, Dual-luminophor pressure-sensitive paint: I. Ratio of reference to sensor giving a small temperature dependency, Sensor. Actuator. B Chem. 97 (2004) 13-21.

[13] K.S. Muhammet Erkan, B.F. Carroll, K.S. Schanze, Preparation and spectroscopic properties of multiluminophore luminescent oxygen and temperature sensor films, Langmuir 21 (2005) 9121-9129.

[14] P. Di, C.D. Jensen, T.J. Juliano, J.W. Gregory, J. Crafton, S. Palluconi, T. Liu, Temperature-compensated fast pressure-sensitive paint, AIAA J. 51 (2013) 2420-2431.

[15] H. Sakaue, K. Miyamoto, T. Miyazaki, A motion-capturing pressure-sensitive paint method, J. Appl. Phys. 113 (2013) 149-156.

[16] Y. Iijima, H. Sakaue, Platinum porphyrin and luminescent polymer for two-color pressure- and temperature-sensing probes, Sensor. Actuat. A Phys. 184 (2012) $128-133$.

[17] Y. Egami, J. Ueyama, S. Furukawa, T. Kameya, Y. Matsuda, H. Yamaguchi, T. Niimi, Development of fast response bi-luminophore pressure-sensitive paint by means of an inkjet printing technique, Meas. Sci. Technol. 26 (2015) 064004.

[18] P. Di, Y. Liu, A grid-pattern PSP/TSP system for simultaneous pressure and temperature measurements, Sensor. Actuator. B Chem. 222 (2015) 141-150.

[19] T. Hyakutake, H. Taguchi, J. Kato, H. Nishide, M. Watanabe, Luminescent multi-layered polymer coating for the simultaneous detection of oxygen pressure and temperature, Macromol. Chem. Phys. 210 (2010) 1230-1234.

[20] K.J. Moon, H. Mori, M. Furukawa, Simultaneous measurement method of pressure and temperature using dual-layer PSP/TSP with lifetime-based method, Meas. Sci. Technol. 29 (2018) 125301.

[21] G. Yu, Z. Wang, X. Qu, Y. Shi, B. Jin, Z. Liu, X. Jin, Effect of polymer matrix on the oxygen pressure sensitivity of pressure sensitive paints, Acta Polym. Sin. 43 (2014) 350-355.

[22] Z. Xie, F. Wang, C.Y. Liu, Organic-inorganic hybrid functional carbon dot gel glasses, Adv. Mater. 24 (2012) 1716-1721.

[23] B. Jin, Y. Li, S. Yi, X. Jin, Effect of polymer matrix on the photophysical behaviors of pressure sensitive films, Polymer 116 (2017) 466-471.

[24] V. Kumar, D.-J. Lee, J.-Y. Lee, Studies of RTV silicone rubber nanocomposites based on graphitic nanofillers, Polym. Test. 56 (2016) 369-378.

[25] Y. Sugioka, D. Numata, K. Asai, S. Koike, K. Nakakita, T. Nakajima, Polymer/ ceramic pressure-sensitive paint with reduced roughness for unsteady measurement in transonic flow, AIAA J. 56 (2018) 1-12.

[26] Y. Sugioka, K. Arakida, M. Kasai, T. Nonomura, K. Asai, Y. Egami, K. Nakakita, Evaluation of the characteristics and coating film structure of polymer/ceramic pressure-sensitive paint, Sensors 18 (2018) 4041.

[27] Y. Wang, K. Wang, Z. Han, Z. Yin, C. Zhou, F. Du, S. Zhou, P. Chen, Z. Xie, High color rendering index trichromatic white and red LEDs prepared from silane-functionalized carbon dots, J. Mater. Chem. C 5 (2017) 9629-9637.

[28] K.J. Mintz, Y. Zhou, R.M. Leblanc, Recent development of carbon quantum dots regarding their optical properties, photoluminescence mechanism, and core structure, Nanoscale 11 (2019) 4634-4652.

[29] D. Yang, S. Guan, Y.g Niu, Z. Xie, S. Zhou, X. Qu, Construction of a hypoxia responsive upconversion nanosensor for tumor imaging by fluorescence resonance energy transfer from carbon dots to ruthenium complex, J. Mater. Chem. B 6 (2018) 2315-2322.

[30] M. Liu, B. Chen, C. Li, C. Huang, Carbon dots: synthesis, formation mechanism, fluorescence origin and sensing applications, Green Chem. 21 (2019) 449-471.

[31] L. Ma, W. Xiang, H. Gao, L. Pei, X. Ma, Y. Huang, X. Liang, Carbon dot-doped sodium borosilicate gel glasses with emission tunability and their application in white light emitting diodes, J. Mater. Chem. C 3 (2015) 6764-6770.

[32] J. Ren, X. Sun, Y. Wang, R. Song, Z. Xie, S. Zhou, P. Chen, Controllable photoluminescent and nonlinear optical properties of polymerizable carbon dots and their arbitrary copolymerized gel glasses, Adv. Optical Matter. 6 (2018) 1701273.

[33] K. Tadanaga, K. Iwashita, T. Minami, N. Tohge, Coating and water permeation properties of $\mathrm{SiO}_{2}$ thin films prepared by the sol-gel method on nylon- 6 substrates, J. Sol. Gel Sci. Technol. 6 (1996) 107-111.

[34] S. Guan, D. Yang, Y. Weng, H. Lu, X. Meng, X. Qu, S. Zhou, Excitation-dependent theranostic nanosheet for cancer treatment, Adv. Healthc. Mater. 7 (2018) 1701123.

[35] S. Guan, L. Wang, S. Xu, D. Yang, G.I.N. Waterhouse, X. Qu, S. Zhou, Vacancyenhanced generation of singlet oxygen for photodynamic therapy, Chem. Sci. 10 (2019) 2336-2341.

[36] Y. Matsuda, K. Uchida, Y. Egami, H. Yamaguchi, T. Niimi, Polymer-particle pressure-sensitive paint with high photostability, Sensors 16 (2016) 550.

[37] F. Wang, Z. Xie, H. Zhang, C.Y. Liu, Y.G. Zhang, Highly luminescent organosilane-functionalized carbon dots, Adv. Funct. Mater. 21 (2011) 1027-1031.

[38] J.W. Gregory, K. Asai, M. Kameda, T. Liu, J.P. Sullivan, A review of pressure-sensitive paint for high-speed and unsteady aerodynamics, Proc. IMechE Part G 222 (2008) 249-290. 